\title{
Polyubiquitylation of Histone H2B
}

\section{Fuqiang Geng and William P. Tansey}

\author{
Cold Spring Harbor Laboratory, Cold Spring Harbor, NY 11724
}

Submitted January 18, 2008; Revised May 6, 2008; Accepted June 5, 2008

Monitoring Editor: Thomas Sommer

\begin{abstract}
Covalent modification of histones by ubiquitylation is a prominent epigenetic mark that features in a variety of chromatin-based events such as histone methylation, gene silencing, and repair of DNA damage. The prototypical example of histone ubiquitylation is that of histone $\mathrm{H} 2 \mathrm{~B}$ in Saccharomyces cerevisiae. In this case, attachment of ubiquitin to lysine 123 (K123) of H2B is important for regulation of both active and transcriptionally silent genes and participates in trans to signal methylation of histone $\mathrm{H} 3$. It is generally assumed that $\mathrm{H} 2 \mathrm{~B}$ is monoubiquitylated at K123 and that it is this single ubiquitin moiety that influences H2B function. To determine whether this assumption is correct, we have re-examined the ubiquitylation status of endogenous $\mathrm{H} 2 \mathrm{~B}$ in yeast. We find that, contrary to expectations, $\mathrm{H} 2 \mathrm{~B}$ is extensively polyubiquitylated. Polyubiquitylation of $\mathrm{H} 2 \mathrm{~B}$ appears to occur within the context of chromatin and is not associated with $\mathrm{H} 2 \mathrm{~B}$ destruction. There are at least two distinct modes of $\mathrm{H} 2 \mathrm{~B}$ polyubiquitylation: one that occurs at K123 and depends on the Rad6-Bre1 ubiquitylation machinery and another that occurs on multiple lysine residues and is catalyzed by an uncharacterized ubiquitin ligase(s). Interestingly, these ubiquitylation events are under the influence of different combinations of ubiquitin-specific proteases, suggesting that they have distinct biological functions. These results raise the possibility that some of the biological effects of ubiquitylation of $\mathrm{H} 2 \mathrm{~B}$ are exerted via ubiquitin chains, rather than a single ubiquitin group.
\end{abstract}

\section{INTRODUCTION}

Over the last decade, it has become apparent that covalent modification of histones plays a prominent role in establishing epigenetic patterns of gene control in eukaryotic cells (Ruthenburg et al., 2007). A variety of histone modifications have been described, including phosphorylation, methylation, and ubiquitylation. These modifications frequently occur in distinct, interrelated patterns, giving rise to the notion that there is a histone code that is read by the cellular machinery to set the transcriptional status of a particular piece of chromatin (Jenuwein and Allis, 2001).

Interestingly, one of the first covalent histone modifications to be discovered was ubiquitylation. In their characterization of the nuclear protein "A24," Busch and colleagues (Goldknopf et al., 1975; Goldknopf and Busch, 1977) described an isopeptide linkage between lysine 119 (K119) of histone $\mathrm{H} 2 \mathrm{~A}$ and the protein that is now known as ubiquitin (Ub). Early studies (e.g., Levinger and Varshavsky, 1982) demonstrated a connection between histone ubiquitylation and the transcriptional status of chromatin, and it is now clear that histone ubiquitylation, which has been reported for H2A, H2B, H3, and H4 (Muratani and Tansey, 2003), is an important regulatory modification involved in both gene silencing (e.g., de Napoles et al., 2004; Fang et al., 2004) and activation (e.g., Kao et al., 2004). Because of the genetics available in yeast, most of what is known about the functional significance of histone ubiquitylation comes from studies of Saccharomyces cerevisiae and histone H2B.

Yeast histone $\mathrm{H} 2 \mathrm{~B}$ is monoubiquitylated at lysine residue 123 (K123) by the Rad6 Ub-conjugating enzyme (Robzyk et

This article was published online ahead of print in $M B C$ in Press (http:/ / www.molbiolcell.org/cgi/doi/10.1091/mbc.E08-01-0050) on June 18, 2008.

Address correspondence to: William P. Tansey (tansey@cshl.edu). al., 2000) and the Bre1 Ub-protein ligase (Hwang et al., 2003; Wood et al., 2003a). Ubiquitylation of H2B (H2B-Ub) at this site participates in a variety of processes, including establishment of cell size (Hwang et al., 2003), meiosis (Robzyk et al., 2000; Yamashita et al., 2004), gene activation (Kao et al., 2004), gene silencing (Dover et al., 2002; Sun and Allis, 2002), and histone H3 methylation (Dover et al., 2002; Sun and Allis, 2002). H2B ubiquitylation is reportedly higher at active genes than at the silenced telomeres (Emre et al., 2005), and both the recruitment and activity of the H2B-ubiquitylation machinery are regulated by interactions with the transcriptional apparatus, including pol II (Xiao et al., 2005), the Paf complex (Ng et al., 2003; Wood et al., 2003b; Xiao et al., 2005), and Bur kinases (Wood et al., 2005). At active genes, H2B ubiquitylation is essential for the processive methylation of histone H3 at lysine residues K4 and K79 (Dover et al., 2002; Sun and Allis, 2002; Dehe et al., 2005; Shahbazian et al., 2005), but not K36 (Li et al., 2003; Wood et al., 2003b), and for recruitment of proteasomal ATPases to chromatin (Ezhkova and Tansey, 2004). Although only $\sim 10 \%$ of steady-state H2B is monoubiquitylated (Robzyk et al., 2000), it is likely that a much larger percentage of H2B is actually ubiquitylated and that deubiquitylation is an active and crucial process (reviewed in Emre and Berger, 2004). In support of this notion, the H2B-Ub-specific protease Ubp8, which is part of the SAGA complex (Henry et al., 2003; Daniel et al., 2004), is important for gene activation (Henry et al., 2003), whereas Ubp10, which is thought to preferentially remove Ub from H2B at the telomeres (Emre et al., 2005; Gardner et al., 2005), is important for gene silencing (Kahana and Gottschling, 1999). These findings have led to a model in which cycles of H2B ubiquitylation and deubiquitylation provide directionality to different stages in the transcription process (e.g., Wyce et al., 2007).

Current understanding of the role of $\mathrm{H} 2 \mathrm{~B}-\mathrm{Ub}$ in transcription, and the search for downstream effector molecules, is predicated on the notion that $\mathrm{H} 2 \mathrm{~B}$ is modified by a single $\mathrm{Ub}$ 
moiety at K123. This notion is based largely on the fact that total $\mathrm{H} 2 \mathrm{~B}$ isolated from yeast cells resolves into two predominant species: the unmodified form ( $\sim 90 \%$; Robzyk et al., 2000) and the form that is monoubiquitylated at K123. It should be noted, however, that the existence of a substantial pool of a ubiquitylated protein such as this is unusual, and in most cases ubiquitylated proteins constitute only a small fraction of the total population of that protein (Kaiser and Tagwerker, 2005). For this reason, detection of $\mathrm{Ub}$ conjugates usually requires methods to specifically enrich for and stabilize ubiquitylated proteins (e.g., Yaglom et al., 1995). Interestingly, we have been unable to find an example in the literature where such directed methods were applied to detect ubiquitylated forms of $\mathrm{H} 2 \mathrm{~B}$. We therefore performed such an analysis to reexamine the ubiquitylation status of endogenous $\mathrm{H} 2 \mathrm{~B}$ in yeast cells. We find that, contrary to expectations, H2B is polyubiquitylated, both at K123 and at multiple other lysine residues in the protein. There are at least two distinct pathways that contribute to $\mathrm{H} 2 \mathrm{~B}$ polyubiquitylation, and these are under the control of distinct deubiquitylating enzymes. These data reveal that previous analyses of the role of ubiquitylation of $\mathrm{H} 2 \mathrm{~B}$ at K123 have been performed within a backdrop of other H2B-ubiquitylation events and raise the possibility that poly-Ub chains mediate the transcriptional effects of $\mathrm{H} 2 \mathrm{~B}-\mathrm{Ub}$.

\section{MATERIALS AND METHODS}

\section{Yeast Strains and Plasmids}

Yeast strains used in this study are listed in Table 1.

The integration plasmid pRS305-HTB1-myc was created by inserting partial HTB1 sequence $(51,+300)$ into the plasmid pRS305; in this case, the HTB1 coding sequence was modified to include a single, carboxy-terminal, mycepitope tag. Plasmid pRS305-HTB1-HA is identical to pRS305-HTB1-myc except that HTB1 carries a single copy of the hemagglutinin (HA)-epitope tag. The plasmids were used to tag endogenous H2B by cutting with HindIII, transforming into the yeast strain JD37-13C (kindly provided by R. Deshaies [California Institute of Technology, Pasadena, CA]; Dohmen et al., 1995), and selecting for the LEU2+ phenotype, which creates strains FGY1 (HTB1-myc) and FGY2 (HTB1-HA). Correct integration was confirmed by PCR and Western blotting (WB).

To express HTB1 from a single-copy plasmid under the control of the endogenous HTB1 promoter, the plasmid p413-HTB1-HA was constructed, which harbors the full HTB1 locus $(-824,+300)$, modified to express H2B containing a single, carboxy-terminal, HA-epitope tag. Sitedirected mutagenesis (Quickchange; Stratagene, La Jolla, CA) was used to create the following H2B mutants in p413-HTB1-HA: 1) K123R, lysine 123 changed to arginine; all other lysine residues intact; 2) K123+, lysine 123 intact and all other lysine residues changed to arginine; 3) K111+, lysine 111 intact and all other lysine residues changed to arginine; 4) $\mathrm{K} 3+$, lysine 3 intact and all other lysine residues changed to arginine; 5) $\mathrm{K} 0$, all lysine residues changed to arginine; 6) NK + , amino terminal lysine residues $\mathrm{K} 3,6,7,11,16$, $17,21,22,30$, and 31 intact and all other lysine residues changed to arginine and 7) coreK+, core lysine residues $34,37,46,49,60,82,88,89$, and 111 intact and all other lysine residues changed to arginine. To express HA-tagged Htb1 proteins-WT and mutant-as the sole source of cellular H2B, a set of yeast strains were made by plasmid shuffle in strain FGY8 ( $\Delta h t b 1 \Delta h t b 2 ;$ Table 1$)$. The ubp 8 and ubp 10 deletion mutants were constructed by replacing the open reading frames (ORFs) with selection marker cassettes using a one-step PCR method (Knop et al., 1999). Deletion of ORFs was confirmed by PCR.

The plasmid pRS426-pCUP1-His7-Ubiquitin ${ }^{\mathrm{G} 76 \mathrm{~A}}$ was constructed by inserting the CUP1 promoter and His7-Ubiquitin (mutant: G76A) coding sequences into pRS426. Plasmids pp389 (YepLac-TRP-pCUP-His6-ubiquitin;G76A), pp390 (YepLac-TRP-pCUP-His6-ubiquitin;G76A K48R), and pp391 (YepLacTRP-pCUP-His6-ubiquitin;G76A K63R) were kindly provided by P. Kaiser (University of California, Irvine, CA; Flick et al., 2004). Plasmid pUB221 (pCUP1-his6-myc-Ubiquitin) was kindly provided by D. Finley (Harvard Medical School, Boston, MA; Yaglom et al., 1995).

To generate His8- and His8-HA-tagged H2B, pRS305-HTB1-HA was modified by site-directed mutagenesis to create pRS305-HTB1-His8 and pRS305HTB1-His8-HA, respectively. The SUMO tagging plasmid pRS306-Flag-SMT3 was constructed by cloning into pRS306 the SMT3 gene with a Flag epitope sequence inserted after the second codon of SMT3. Endogenous HTB1 was then C-terminally HA-, his8-HA-, or his8-tagged by integrating the appropriate HindIII-digested plasmid and selecting for LEU2+. Endogenous SMT3 (encoding SUMO) was N-terminally Flag-tagged via a two-step gene replacement with PmII-digested pRS306-Flag-SMT3. Correction integration was confirmed by PCR and Western blot.

\section{Analysis of $\mathrm{H} 2 \mathrm{~B}$ Ubiquitylation and Stability}

Ubiquitylation assays were carried out as described (Muratani et al., 2005) with some modification. Briefly, in the indicated strains, poly-histidinetagged $\mathrm{Ub}$ was induced by addition of $\mathrm{CuSO}_{4}$ to a final concentration $0.2 \mathrm{mM}$. Cells were collected, disrupted by glass bead beating in buffer A2 (6 M guanidine- $\mathrm{HCl}, 100 \mathrm{mM} \mathrm{Na}{ }_{2} \mathrm{HPO}_{4} / \mathrm{NaH}_{2} \mathrm{PO}_{4}, \mathrm{pH} 8.0,10 \mathrm{mM}$ imidazole, 250 $\mathrm{mM} \mathrm{NaCl}$, and $0.5 \% \mathrm{NP}-40$ ), and soluble material was recovered. At this point, $90 \%$ of the material was incubated with Ni-NTA agarose resin (Qiagen, Chatsworth, CA) for $2 \mathrm{~h}$ at $4^{\circ} \mathrm{C}$. The resin was collected, washed twice with buffer A2, once with buffer A2/T2 (1 vol buffer A2 and 3 vol buffer T2: $50 \mathrm{mM}$ $\mathrm{Na}_{2} \mathrm{HPO}_{4} / \mathrm{NaH}_{2} \mathrm{PO}_{4}, \mathrm{pH} 8.0,250 \mathrm{mM} \mathrm{NaCl}, 20 \mathrm{mM}$ imidazole, $0.5 \%$ NP-40) and twice with Buffer T2. Beads were collected and ubiquitylated proteins eluted with $2 \times$ SDS loading buffer containing $250 \mathrm{mM}$ imidazole. Total proteins were recovered from the remaining $10 \%$ of the initial lysate (input) by ethanol precipitation, and resuspended in $1 \times$ SDS loading buffer. Proteins were then resolved by SDS-PAGE and analyzed by WB, using anti-HA

Table 1. Yeast strains used in this study

\begin{tabular}{|c|c|c|}
\hline Strain & Genotype & Source \\
\hline JD47-13C & 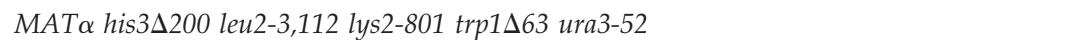 & Dohmen et al. (1995) \\
\hline FGY1 & $=\mathrm{JD} 47-13 \mathrm{C}$, except HTB1-myc::LEU2 & This study \\
\hline FGY2 & $=$ JD47-13C, except HTB1-HA::LEU2 & This study \\
\hline FG4742 & $M A T \alpha$ his $\Delta 1$ leu $2 \Delta 0$ lys $2 \Delta 0$ ura $3 \Delta 0$ & Openbiosystem \\
\hline FGY3 & $=\mathrm{BY} 4742$, except rad6::KanMX & Openbiosystem \\
\hline FGY4 & $=\mathrm{BY} 4742$, except bre $1::$ KanMX & Openbiosystem \\
\hline FGY5 & $=\mathrm{BY} 4742$, except $u b p 8:: L E U 2$ & This study \\
\hline FGY6 & $=\mathrm{BY} 4742$, except $u b p 10:: \operatorname{KanMX}$ & This study \\
\hline FGY7 & $=\mathrm{BY} 4742$, except $u b p 8::$ LEU2 ubp10::KanMX & This study \\
\hline JR5-2A & MATa htb-1-1 htb2-1 leu2-3,-112 his3-11,-15 trp1-1 ura3-1 ade2-1 can1-100 〈Ycp50-HTB1 $\rangle$ & Recht and Osley (1999) \\
\hline FGY8 & $=J R 5-2 A$, except hta2-htb2::KanMX & This study \\
\hline FGY9 & $=$ FGY8, except bre $1:: L E U 2$ & This study \\
\hline FGY10 & $=$ FGY 8 , except $u b p 8:: L E U 2$ & This study \\
\hline FGY11 & $=$ FGY 8, except $u b p 10:: T R P 1$ & This study \\
\hline FGY12 & $=$ FGY 8, except $u b p 8:: L E U 2$ ubp $10:: T R P 1$ & This study \\
\hline FGY13 & $=\mathrm{BY} 4742$, except HTB1-HA $:: L E U 2$ & This study \\
\hline FGY14 & $=\mathrm{BY} 4742$, except HTB1-His8 $:: L E U 2$ & This study \\
\hline FGY15 & $=\mathrm{BY} 4742$, except HTB1-His8-HA::LEU2 & This study \\
\hline FGY16 & $=\mathrm{BY} 4742$, except HTB1-His8-HA::LEU2 Flag-SMT3 & This study \\
\hline FGY17 & $=\mathrm{BY} 4742$, except Flag-SMT3 & This study \\
\hline
\end{tabular}


HRP-conjugated antibody (Roche, Nutley, NJ). To assay the association of ubiquitylated H2B with chromatin, a similar approach was used, except that cellular material was first fractionated as described (Liang and Stillman, 1997). The Orc3 antibody was a gift from B. Stillman (Cold Spring Harbor Laboratory; Liang and Stillman, 1997).

Pulse-chase analysis was performed as described (Salghetti et al., 2000).

\section{RNA Analyses}

For GAL gene induction, yeast were grown overnight in rich media with $2 \%$ raffinose to an $\mathrm{OD}_{600}$ of $\sim 0.5$ and were induced by addition of $2 \%$ galactose. Cells were collected after $1 \mathrm{~h}$ of induction and total RNA extracted with hot phenol. Random hexamer-primed reverse-transcription was performed with the TaqMan Reverse-Transcription Kit (Applied Biosystems, Foster City, CA). Real-time PCR was performed using an Opticon (Bio-Rad, Richmond, CA) thermocycler and the Cyber Green PCR Kit (Applied Biosystems). Genespecific primers for GAL10 and 25s rRNA have been described (Muratani et al., 2005).

\section{RESULTS}

\section{H2B Is Polyubiquitylated}

It is commonly accepted that, in budding yeast, histone $\mathrm{H} 2 \mathrm{~B}$ is modified by covalent attachment of a single $\mathrm{Ub}$ moiety to lysine residue 123 . This notion is based largely on the observation that $\sim 10 \%$ of $\mathrm{H} 2 \mathrm{~B}$ is present in the monoubiquitylated form in extracts from yeast cells (Robzyk et al., 2000). It should be noted, however, that this extent of ubiquitylation is unusual and that Ub-modified proteins are typically present in much lower amounts because of active deubiquitylation or proteasomal proteolysis. For the most part, therefore, ubiquitylated proteins can only efficiently be detected when they are selected for under conditions that block $\mathrm{Ub}$ removal and/or destruction by the proteasome. In our survey of the literature, we were unable to find an example of where this approach had been applied to histone H2B. Because models for how $\mathrm{H} 2 \mathrm{~B}-\mathrm{Ub}$ functions are different when considering mono- versus polyubiquitylation or a single versus multiple sites of $\mathrm{Ub}$ conjugation, we used a directed method to characterize H2B ubiquitylation in exponentially growing yeast cells.

To detect $\mathrm{H} 2 \mathrm{~B}-\mathrm{Ub}$ conjugates, we used an approach in which polyhistidine-tagged $\mathrm{Ub}$ was expressed in yeast (bearing HA epitope-tagged H2B) under the control of the copper-inducible CUP1 promoter (Yaglom et al., 1995). In this instance, the tagged $\mathrm{Ub}$ also carried a mutation (G76A) that prevents $\mathrm{Ub}$ removal by $\mathrm{Ub}$-specific proteases (Hodgins et al., 1992). Total ubiquitylated proteins were purified under denaturing conditions by nickel-affinity (Ni-NTA) chromatography, and H2B-HA was detected by Western blotting (Figure 1). As expected (Robzyk et al., 2000), analysis of total $\mathrm{H} 2 \mathrm{~B}-\mathrm{HA}$ (input) revealed the presence of two predominant $\mathrm{H} 2 \mathrm{~B}$ species, one corresponding to free $\mathrm{H} 2 \mathrm{~B}$ and the other corresponding to the monoubiquitylated form. Analysis of $\mathrm{H} 2 \mathrm{~B}-\mathrm{HA}$ present in the Ni-NTA-purified material, in contrast, revealed a ladder of high-molecular-weight H2B conjugates (lane 3) that began at a size corresponding to monoubiquitylated H2B ( $\sim 25 \mathrm{kDa})$ and extended upward. At least nine distinct species could be resolved under these conditions, consistent with the notion that H2B is modified by addition of multiple $\mathrm{Ub}$ moieties. Although the relative levels of mono- versus polyubiquitylated H2B cannot be estimated from analysis of the Ni-NTA-purified material, we do note that a species corresponding in molecular weight to doubly ubiquitylated $\mathrm{H} 2 \mathrm{~B}$ can be detected in total extracts of cells expressing the noncleavable $\mathrm{Ub}$ (input; visible in the bracketed $\mathrm{H} 2 \mathrm{~B}-\mathrm{Ub}$ region in lanes $3-5$ ), suggesting that at a significant portion of $\mathrm{H} 2 \mathrm{~B}$ has at least two $\mathrm{Ub}$ groups attached. This conclusion was validated by purification of endogenous, polyhistidine-tagged H2B (Supplemental Fig-

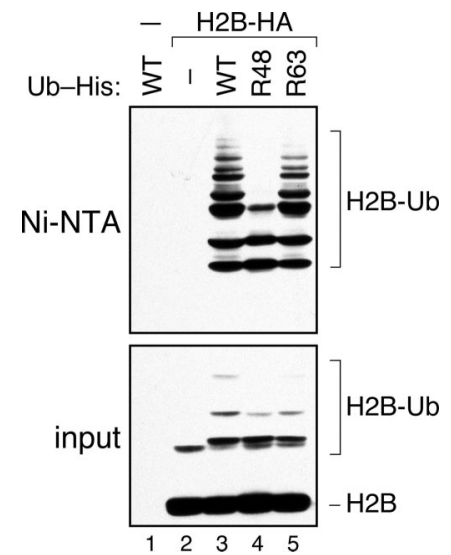

Figure 1. Histone H2B is polyubiquitylated. Yeast cells were engineered to express either His-tagged WT or mutant (K48R or K63R) nonremovable (G76A) ubiquitin, together with either myc-tagged (FGY1; lane 1) or HA-epitope-tagged H2B (FGY2; lanes 2-5). Cells were lysed, and HA-tagged proteins present in the total lysate (input) or His-purified (Ni-NTA) material were detected by WB. The position of free H2B is indicated, as is the position of the $\mathrm{H} 2 \mathrm{~B}-\mathrm{Ub}$ conjugates. Note that the monoubiquitylated form of H2B-HA appears as a doublet in the input; we presume that this corresponds to H2B-HA that is monoubiquitylated with either endogenous $\mathrm{Ub}$ (bottom panel) or the slightly larger His-Ub.

ure S1); under these conditions, we could detect di- and triubiquitylated H2B in the absence of overexpressed, nonremovable $\mathrm{Ub}$.

To determine whether the high-molecular-weight H2B-HA conjugates are modified by poly-Ub chains, we expressed mutant forms of polyhistidine-tagged $\mathrm{Ub}$ (G76A) in which one of the two prominent sites of Ub-chain formation- $\mathrm{K} 48$ or K63 (Pickart and Fushman, 2004)—was mutated to arginine. This analysis revealed that expression of the K48R mutant $\mathrm{Ub}$ reduced the number of $\mathrm{H} 2 \mathrm{~B}-\mathrm{Ub}$ species to approximately three (lane 4), whereas expression of the K63 mutant (lane 5) had little if any effect on the extent of H2B modification. These data reveal that H2B is modified by at least one, and possibly more, K48-linked polyubiquitin chains and that K63-linked chains are unlikely to contribute significantly to the pool of ubiquitylated $\mathrm{H} 2 \mathrm{~B}$.

\section{There Are at Least Two Distinct Types of Polyubiquitylated $\mathrm{H} 2 \mathrm{~B}$}

We next asked whether the poly-Ub chains associated with $\mathrm{H} 2 \mathrm{~B}$ were linked to the canonical lysine 123, other lysine residues in the protein, or both. We first used the His- $\mathrm{Ub}$ assay to examine the poly- $\mathrm{Ub}$ status of a mutant $\mathrm{H} 2 \mathrm{~B}$ in which K123 was changed to arginine (K123R; Figure 2A). As expected (Robzyk et al., 2000), the K123R mutation strongly reduced the levels of monoubiquitylated $\mathrm{H} 2 \mathrm{~B}$ present in total cell lysates (input; cf. lanes 1 and 2). Analysis of the Ni-NTA-purified material, in contrast, revealed that a significant pool of multiply ubiquitylated H2B remained in the K123R mutant, suggesting that residues in addition to K123 are sites for $\mathrm{H} 2 \mathrm{~B}$ ubiquitylation. Note, however, that total levels of multiply ubiquitylated $\mathrm{H} 2 \mathrm{~B}-\mathrm{HA}$ are reduced in the K123R mutant, consistent with the notion that lysine 123 might also be polyubiquitylated.

We further probed the nature of $\mathrm{H} 2 \mathrm{~B}-\mathrm{Ub}$ by examining the ubiquitylation status of four additional $\mathrm{H} 2 \mathrm{~B}$ mutants in which groups of lysine residues were retained, whereas other lysine residues were changed to arginine: 1) NK+, 
A

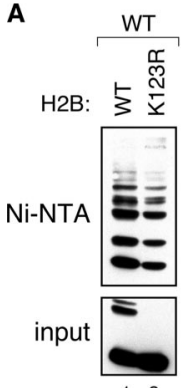

B

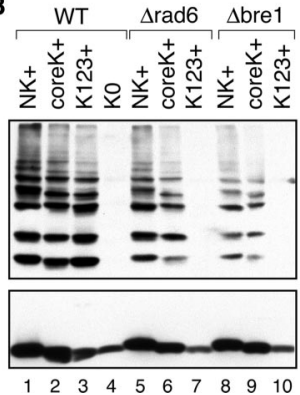

C

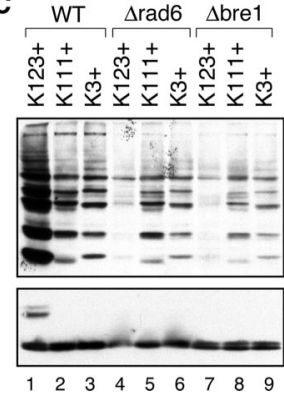

Figure 2. There are at least two distinct pathways of H2B polyubiquitylation. Ubiquitylation of the indicated HA-tagged $\mathrm{H} 2 \mathrm{~B}$ mutants was measured as described in the legend to Figure 1, except that H2B-HA was expressed from a centromeric plasmid in strain BY4742. (A) The K123R H2B mutant is polyubiquitylated. (B) Ubiquitylation at lysine 123 depends exclusively on Rad6-Bre1. The ubiquitylation status of four different $\mathrm{H} 2 \mathrm{~B}$ mutants- $\mathrm{NK}+$, core + , $\mathrm{K} 123+$, and $\mathrm{K} 0$ (described in the text)-was determined in either WT cells (lanes 1-4), or congenic strains in which RAD6 (FGY3; lanes 5-7) or BRE1 (FGY4; lanes 8-10) were deleted. (C) H2B proteins carrying single lysine residues are polyubiquitylated. The ubiquitylation status of $\mathrm{K} 123+, \mathrm{K} 111+$, and $\mathrm{K} 3+\mathrm{H} 2 \mathrm{~B}$ mutants was examined in WT (lanes 1-3), $\Delta$ rad6 (lanes 4-6), and $\Delta b r e 1$ (lanes 7-9) cells. Note that a more sensitive form of detection was used in the experiment in $\mathrm{C}$ than in $\mathrm{B}$, because of the lower absolute signal from the single lysine $\mathrm{K} 111+$ and $\mathrm{K} 3+$ mutants. For this reason, monoubiquitylated $\mathrm{K} 123+\mathrm{H} 2 \mathrm{~B}$ is visible in the input in $\mathrm{C}$ (cf. lanes in $\mathrm{B}$ with lane 1 in $\mathrm{C})$.

which retained lysine residues in the amino-terminal portion of $\mathrm{H} 2 \mathrm{~B}(\mathrm{~K} 3,6,7,11,16,17,21,22,30,31)$; 2) coreK+, with lysine residues 34, 37, 46, 49, 60, 82, 88, 89, and 111 intact; 3) $\mathrm{K} 123+$, where $\mathrm{K} 123$ is the sole lysine in the protein; and 4) $\mathrm{K} 0$, a lysine-free mutant of $\mathrm{H} 2 \mathrm{~B}$. This analysis revealed that, with the exception of the $\mathrm{K} 0$ protein (Figure 2B; lane 4), all three lysine-containing H2B mutants were multiply ubiquitylated (lanes 1-3). We conclude from this result that $\mathrm{H} 2 \mathrm{~B}$ is not subject to extensive lysine-free ubiquitylation (e.g., Bloom et al., 2003) and that multiple lysine residues within H2B can serve as sites of conjugation for $\mathrm{Ub}$. Consistent with the results of the experiment presented in Figure $2 \mathrm{~A}$, this result also demonstrates that K123 can itself be subject to polyubiquitylation.

To examine the contribution of the previously described Rad6-Bre1 Ub-ligase pathway (Robzyk et al., 2000; Hwang et al., 2003; Wood et al., 2003a) to these ubiquitylation events, we also examined each mutant H2B in congenic $\Delta$ rad6 and $\Delta b r e 1$ strains (lanes 5-10). We found that polyubiquitylation of the $\mathrm{NK}+$ and coreK + mutants was largely insensitive to disruption of either RAD6 or BRE1, revealing that this pathway is not responsible for ubiquitylation of these proteins. For the $\mathrm{K} 123$ + mutant, however, ubiquitylation was dependent on both RAD6 and BRE1: the K123+ protein in the $\Delta$ rad6 and $\Delta b r e 1$ strains was largely indistinguishable from the $\mathrm{K} 0$ mutant. This finding reveals that both mono- and polyubiquitylation of H2B at K123 is dependent on Rad6Bre1 and that there must be at least one other Ub-ligase system responsible for the ubiquitylation of the other $\mathrm{H} 2 \mathrm{~B}$ lysine residues.

We subsequently analyzed a panel of mutant $\mathrm{H} 2 \mathrm{~B}$ proteins, each of which contained a single lysine residue. The majority of these mutants were ubiquitylated (not shown), and we therefore selected two for further analysis: K111+ and $\mathrm{K} 3+$. We selected these two mutants because they carried lysine residues in different domains of the protein (K111+ in the core, $\mathrm{K} 3+$ in the amino terminus). This anal-

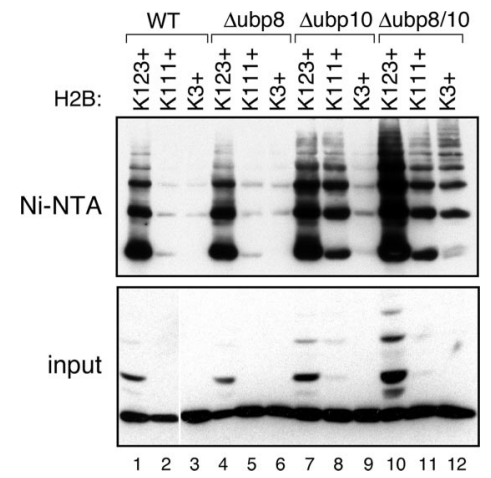

Figure 3. Deubiquitylating enzymes differentially affect Ub-chain length at particular H2B lysine residues. Ubiquitylation of the indicated HA-tagged H2B mutants was measured as described in the legend to Figure 1, except that H2B-HA was expressed from a centromeric plasmid in BY4742. Note also that to detect the effects of deleting UBP8 and/or UBP10, we used a cleavable form of His-Ub in which residue G76 of $\mathrm{Ub}$ is intact (Yaglom et al., 1995). Four congenic yeast strains were analyzed: WT (BY4742; lanes 1-3), $\Delta u b p 8$ (FGY5; lanes 4-6), $\Delta u b p 10$ (FGY6; lanes 7-9), and $\Delta u b p 8 /$ $\Delta$ ubp10 (FGY7; lanes 10-12).

ysis is shown in Figure 2C. Both the $\mathrm{K} 111+$ and $\mathrm{K} 3+$ mutants were polyubiquitylated (lanes 2 and 3), although to a significantly less extent than K123+ (lane 1). In all both cases, loss of either Rad6 or Bre1 did not eliminate polyubiquitylation (cf. lanes 1-3 with 4-9), although some small differences were observed in the various mutants (e.g., the $\mathrm{K} 111+$ mutant in the $\Delta b r e 1$ strain; lane 8). This result emphasizes that ubiquitylation events at K123 are uniquely dependent on the Rad6-Bre1 proteins, whereas ubiquitylation at other sites within $\mathrm{H} 2 \mathrm{~B}$ must be directed by a distinct Ub-ligase system(s).

Histone $\mathrm{H} 2 \mathrm{~B}$ deubiquitylation plays an important role in mediating the transcriptional effects of $\mathrm{H} 2 \mathrm{~B}$ ubiquitylation (Emre and Berger, 2004). Two deubiquitylating enzymesUbp8 (Henry et al., 2003; Daniel et al., 2004) and Ubp10 (Emre et al., 2005; Gardner et al., 2005) - have been shown to deubiquitylate $\mathrm{H} 2 \mathrm{~B}$ at $\mathrm{K} 123$. The finding that other sites in H2B are ubiquitylated and that K123 can be polyubiquitylated raises the possibility that these noncanonical ubiquitylation events can also be regulated by Ubp8 and/or Ubp10. We therefore examined the ubiquitylation status of three single lysine $\mathrm{H} 2 \mathrm{~B}$ mutants (K123+, $\mathrm{K} 111+$, and $\mathrm{K} 3+$ ) in $\Delta u b p 8, \Delta u b p 10$, and $\Delta u b p 8 / \Delta u b p 10$ yeast strains to compare how different sites of $\mathrm{H} 2 \mathrm{~B}$ ubiquitylation respond to loss of these deubiquitylating enzymes (Figure 3). In this analysis, the $\mathrm{K} 123+$ mutant responded little if any to loss of UBP8 (cf. lanes 1 and 4), whereas deletion of UBP10 increased the ubiquitylation levels of this protein considerably (lane 7). The failure of $\Delta u b p 8$ to affect $\mathrm{K} 123+$ ubiquitylation appears to be the result of redundancy between Ubp8 and Ubp10, because simultaneous disruption of both proteins increased the levels of mono- and polyubiquitylated $\mathrm{K} 123+$ considerably (lane 10). This result is consistent with previous observations showing that loss of Ubp8 and Ubp10 synergize to promote ubiquitylation of WT H2B (Gardner et al., 2005). Importantly, because the $\Delta u b p 8 / \Delta u b p 10$ mutant accumulates significant levels of poly ubiquitylated K123+ H2B (visible in both the input and Ni-NTA-purified material), this result also reveals that these two deubiquitylating enzymes also play a role in mediating the extent of Ub-chain formation at lysine residue 123. 
Analysis of the $\mathrm{K} 3+$ mutant revealed a similar pattern of behavior as K123+ (cf. lanes 3, 6, 9, and 12), suggesting that Ubp 8 and Ubp10 both work to limit the extent of ubiquitylation at this site. Intriguingly, however, analysis of the $\mathrm{K} 111+$ mutant revealed a different pattern of response to deletion of UBP8 and UBP10. Like the $\mathrm{K} 123+$ mutant, deletion of UBP8 had little effect on the levels of ubiquitylated $\mathrm{K} 111+$ (cf. lanes 2 and 5), whereas deletion of UBP10 increased the extent of $\mathrm{K} 111+$ ubiquitylation considerably (lane 8). Unlike the K123+ mutant, however, there appeared to be little redundancy between $\mathrm{Ubp} 8$ and $\mathrm{Ubp} 10$, because the ubiquitylation status of the K111+ mutant was very similar in the $\Delta u b p 8$ and $\Delta u b p 8 / \Delta u b p 10$ strains (cf. lanes 8 and 11). From this result we conclude that ubiquitylation at lysine 111 of $\mathrm{H} 2 \mathrm{~B}$ is predominantly under the control of Ubp10 and that Ubp8 does little to limit Ub-chain formation at this site. The differential behavior of the K111+ mutant demonstrates that deubiquitylating enzymes Ubp8 and Ubp10 do not work globally on ubiquitylated H2B, but display specificity for particular sites of Ub conjugation. This finding further supports the idea that multiple pathways regulate $\mathrm{H} 2 \mathrm{~B}$ ubiquitylation and suggests that ubiquitylation, and deubiquitylation, of different lysine residues within H2B may mediate distinct functional outcomes.

\section{Polyubiquitylated H2B Is Chromatin-associated}

A central factor in models for how ubiquitylation of $\mathrm{H} 2 \mathrm{~B}$ at K123 controls gene expression is that H2B-Ub is incorporated into chromatin and acts to either recruit factors to sites of gene regulation or to disrupt local chromatin structure (Osley, 2004). It is conceivable, however, that the additional ubiquitylated forms of $\mathrm{H} 2 \mathrm{~B}$ that we detect do not exist within the context of chromatin, in which case a role for these modifications in the control of chromatin-mediated events would be more difficult to imagine. We therefore asked whether forms of $\mathrm{H} 2 \mathrm{~B}$ with noncanonical ubiquitin chains can associate with chromatin, as shown in Figure 4. We measured the ubiquitylation status of the $\mathrm{NK}+$, coreK+, and $\mathrm{K} 123+\mathrm{H} 2 \mathrm{~B}$ mutants after fractionation of chromatin using the method of Liang and Stillman (1997). In this assay, total cell extracts are first subject to low-speed centrifugation to separate soluble proteins from the insoluble matrix and chromatin. The insoluble material is then treated with micrococcal nuclease to release chromatin from this fraction, leading to the appearance of chromatin-associated proteins in the supernatant after a second round of centrifugation. The DNA replication factor Orc3 was our control in this assay (Liang and Stillman, 1997). As shown in the figure, ubiquitylated forms of all three $\mathrm{H} 2 \mathrm{~B}$ mutants were present in the insoluble material (pellet) after the first round of centrifugation (lanes 3, 8, and 13), and all three could be released from the pellet by treatment with micrococcal nuclease, to an extent similar to that observed for total levels of Orc3 (lanes 4, 9, and 14). From this result, we conclude that a significant portion of K123-ubiquitylated $\mathrm{H} 2 \mathrm{~B}$, as well as H2B ubiquitylated at other sites, is tightly associated with chromatin. Interestingly, we also observed that ubiquitylated forms of H2B were generally released from chromatin more efficiently than the nonubiquitylated species (cf. NiNTA vs. input for lanes 4 and 5, 9 and 10, and 14 and 15), suggesting that chromatin containing ubiquitylated $\mathrm{H} 2 \mathrm{~B}$ may be more accessible than that containing the unmodified form.

\section{Ubiquitylation of Lysine 123 of $\mathrm{H} 2 \mathrm{~B}$ Can Be Essential}

Rad6-Bre1-mediated ubiquitylation of histone H2B at K123 is a nonessential process in yeast. Yeast deleted for RAD6 or

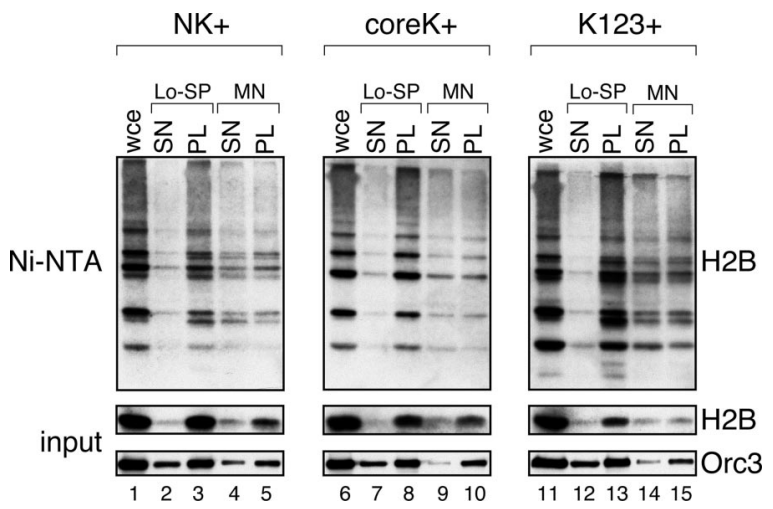

Figure 4. Ubiquitylated $\mathrm{H} 2 \mathrm{~B}$ is associated with chromatin. Wildtype yeast cells (JR5-2A) were engineered by plasmid shuffle to express the indicated H2B-HA mutants (from a centromeric plasmid) as well as His-tagged G76A ubiquitin. Chromatin fractionation was then performed as described (Liang and Stillman, 1997). Whole cell extracts (WCE; lanes 1, 6, and 11) were subject to a low-speed centrifugation (Lo-SP) and separated into soluble material (SN; lanes 2, 7, and 12) and an insoluble pellet containing chromatin (PL; lanes 3, 8, and 13). The insoluble material was then treated with micrococcal nuclease (MN) to release mono- and polynucleosomes into the soluble fraction. These extracts were then subject to a second round of centrifugation to separate remaining insoluble material (PL; lanes 5, 10, and 15) from the solubilized nucleosomes (SN; lanes 4, 9, and 14). Nickel-affinity chromatography was then used to isolate ubiquitylated proteins in each fraction. H2B-HA in the Ni-NTA and input material, as well as Orc3 (control) in the input, were detected by WB.

$B R E 1$, or carrying the K123R H2B mutant, display reduced GAL gene activation (Kao et al., 2004), as well as defects in meiosis (Robzyk et al., 2000; Yamashita et al., 2004), gene silencing (Dover et al., 2002; Sun and Allis, 2002), and histone H3 methylation (Dover et al., 2002; Sun and Allis, 2002), but are nonetheless viable. Our finding that there are multiple ubiquitylation events on histone $\mathrm{H} 2 \mathrm{~B}$ raises the important point that previous analyses of the functional consequences of Rad6-Bre1-dependent H2B ubiquitylation (at K123) have been performed within a backdrop of other H2B-ubiquitylation events, which in turn may mask effects resulting from loss of Rad6-Bre1 ubiquitylation. Although we do not know the identity of the cellular machinery that ubiquitylates the noncanonical sites on $\mathrm{H} 2 \mathrm{~B}$, our finding that ubiquitylation of the K123+ mutant entirely dependent on Rad6-Bre1 (Figure 2) gives us the opportunity to examine the contribution of this site of ubiquitylation in isolation.

We therefore asked whether $\mathrm{H} 2 \mathrm{~B}$ with a single lysine at position 123 would support GAL gene activation and yeast cell viability, and-if so-whether this was dependent on Bre1. We examined galactose-mediated induction of the GAL10 gene (Figure 5A). Consistent with previous reports (Henry et al., 2003; Kao et al., 2004), we found that the K123R mutation reduced GAL10 induction about threefold. Under identical conditions, however, the $\mathrm{K} 123+$ mutant showed little if any induction of GAL10, arguing that other lysine residues within $\mathrm{H} 2 \mathrm{~B}$ (besides $\mathrm{K} 123$ ) are important for GAL activation. Although this result itself is not informative, it does contrast strongly with the effects of these mutations on cell viability (Figure 5B), as described below.

We used a plasmid shuffle assay to compare the ability of wild-type (WT), K123R, K123+, and $\mathrm{K} 0 \mathrm{H} 2 \mathrm{~B}$ proteins to support yeast growth, both in the presence and absence of BRE1 (Figure 5B). As expected, the WT and K123R H2B proteins were functional in this assay and were not affected 
A
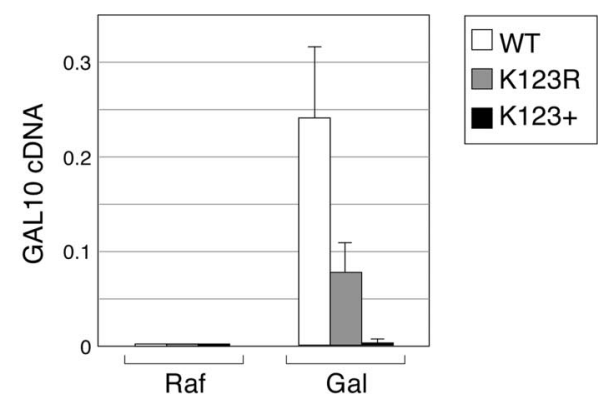

Figure 5. Ubiquitylation at lysine 123 of $\mathrm{H} 2 \mathrm{~B}$ can be essential for viability. Plasmid shuffle was used to express $\mathrm{WT} \mathrm{H} 2 \mathrm{~B}$ - and the $\mathrm{K} 123 \mathrm{R}$ and $\mathrm{K} 123+$ mutants-as the sole source of histone $\mathrm{H} 2 \mathrm{~B}$ in strains FGY8, FGY9, and FGY10 (Table 1). (A) Lysine 123 of $\mathrm{H} 2 \mathrm{~B}$ is required for full GAL gene induction, but is not sufficient. Yeast were grown in raffinose and either uninduced (Raf) or induced with galactose (Gal) for 60 min. RNA was collected and reverse-transcribed into cDNA, and levels of GAL10 and 25S rRNA cDNAs were determined by quantitative PCR. "GAL10 cDNA" is expressed relative to the $25 S$ rRNA control. (B) The $K 123+$ and $\Delta b r e 1$ mutations are synthetically lethal. The indicated $\mathrm{H} 2 \mathrm{~B}$ proteins were expressed in either WT (BRE1; FGY8) or $\Delta b r e 1$ (FGY9) cells, together with a WT H2B protein expressed from a plasmid bearing the $U R A 3$ gene. Yeast were struck onto media containing 5-fluoroorotic acid (FOA) to eliminate cells expressing WT H2B. The K123+ H2B mutant supports viability in the BRE1, but not $\triangle b r e 1$, cells. (C) No genetic interaction between $\mathrm{K} 123+$ and $\Delta u b p 8$. Assay performed as in B but with UBP8 (FGY8) and $\Delta u b p 8$ (FGY10) cells. (D) No genetic interaction between K123+ and $\Delta u b p 10$. Assay performed as in B but with UBP10 (FGY8) and $\Delta u b p 10$ (FGY11) cells. (E) The K123+ and $\Delta u b p 8 / \Delta u b p 10$ mutations are synthetically lethal. Assay performed as in B but with UBP8/10 (FGY8) and $\Delta u b p 8 / \Delta u b p 10$ (FGY12) cells.

\section{B}

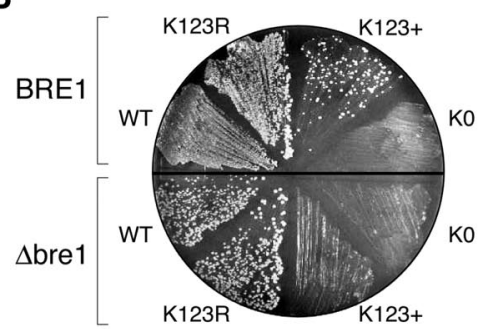

D

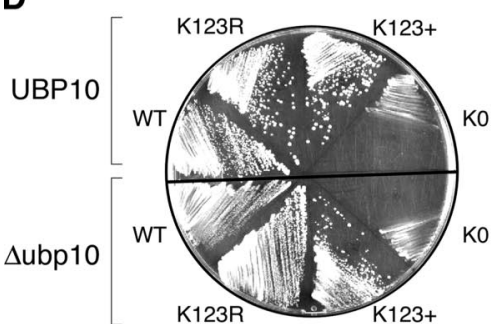

C

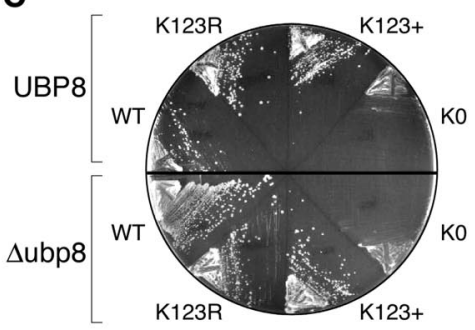

E

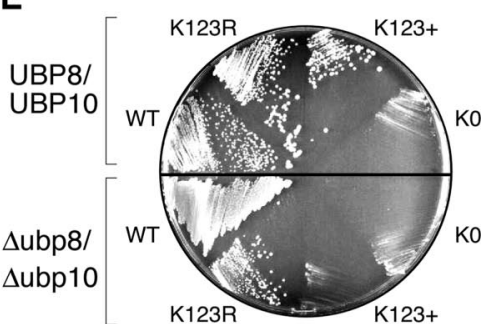

by loss of BRE1. The lysine-free H2B protein (K0), in contrast, did not support viability in either strain background. Interestingly, however, H2B bearing only a single lysine at position $123(\mathrm{~K} 123+)$ did allow yeast growth in BRE1 cells, albeit at a reduced level compared with the WT protein. Moreover, the ability of the K123+ mutant to support cell growth was dependent on BRE1, as the K123+/Dbre1 combination was inviable. We conclude from this result that most of the lysine residues in H2B are dispensable for the essential functions of this histone and that in the absence of these lysine residues ubiquitylation at K123 is an essential process.

Because of the role for histone deubiquitylation in mediating the transcriptional effects of $\mathrm{H} 2 \mathrm{~B}-\mathrm{Ub}$ (Emre and Berger, 2004), we repeated the above analysis, but this time in cells that either expressed or lacked Ubp8 (Figure 5C), Ubp10 (Figure 5D), or both (Figure 5E). Deletion of either UBP8 or UBP10 was not synthetic with the K123+ H2B mutation. Remarkably, however, the double $u b p 8 / \Delta u b p 10$ deletion was synthetically lethal with the K123+ mutation. This result reveals that deubiquitylation of H2B at K123, like ubiquitylation itself, can be an essential process.

\section{DISCUSSION}

Analysis of $\mathrm{H} 2 \mathrm{~B}$ ubiquitylation in the genetically tractable yeast $S$. cerevisiae has been instrumental in revealing the extent to which this process can regulate gene activity. Exactly how ubiquitylation regulates H2B function is, however, unknown. To understand this mechanism, it is essential to understand the nature of how H2B is modified by ubiquitin. We used a directed method to examine the ubiq- uitylation status of $\mathrm{H} 2 \mathrm{~B}$, and various mutants, in yeast cells. We found that, contrary to expectations, $\mathrm{H} 2 \mathrm{~B}$ is subject to extensive polyubiquitylation, both at the canonical K123 residue (which is assumed to be monoubiquitylated) and at multiple other sites within the protein. Ubiquitylation at K123 depends on the characterized Rad6-Bre1 pathway, whereas ubiquitylation at other sites occurs independent of Rad6 or Bre1. The existence of these multiple ubiquitylation events challenges the notion that a single $\mathrm{Ub}$ moiety is responsible for the biological activities of $\mathrm{H} 2 \mathrm{~B}-\mathrm{Ub}$ and suggests that poly-Ub chains, perhaps attached to multiple sites on $\mathrm{H} 2 \mathrm{~B}$, may influence its function.

Our studies have revealed two previously unrecognized types of $\mathrm{H} 2 \mathrm{~B}$ ubiquitylation: ubiquitylation at noncanonical lysine residues (i.e., not K123), and polyubiquitylation at lysine 123. Using "chain terminating" forms of Ub (Figure 1 ), we have shown that most of these polyubiquitylation events involve $\mathrm{K} 48$-linked $\mathrm{Ub}$ chains and that there are multiple sites of Ub-chain formation within $\mathrm{H} 2 \mathrm{~B}$. Analysis of single lysine H2B mutants reveals that most of the lysine residues within the protein can be subject to some ubiquitylation (Figure 2 and data not shown), suggesting that there are either multiple pathways directing ubiquitylation at the various sites on $\mathrm{H} 2 \mathrm{~B}$ or that a smaller number of pathways can ubiquitylate $\mathrm{H} 2 \mathrm{~B}$ without targeting selected lysine residues. The latter scenario is particularly common in the $\mathrm{Ub}$ proteasome system, where finding specific lysine residues required for $\mathrm{Ub}$ conjugation is the exception, rather than the rule (e.g., Crook et al., 1996). It is important to note, however, that loss of Rad6 or Bre1 has little effect on the ubiquitylation status of $\mathrm{NK}+$, coreK,$+ \mathrm{K} 111+$, and $\mathrm{K} 3+$ mutants (Figure 2), revealing that 1) Rad/Bre1 is fairly specific to K123 and 2) 
at least one other Ub-ligase must mediate ubiquitylation events at the other sites. It is also important to note that although the other Ub-ligase(s) may not have specificity toward particular lysine residues, the output of its activity could be very specific, because Ubp 8 and Ubp10 appear to have different preferences for removing $\mathrm{Ub}$ from select lysine residues (Figure 3 ).

What is the function of these noncanonical K48-linked polyubiquitin chains? Although K48-linked chains are typically implicated in proteolysis (Pickart and Fushman, 2004), we think it is unlikely that these modifications direct $\mathrm{H} 2 \mathrm{~B}$ destruction. Histones, including $\mathrm{H} 2 \mathrm{~B}$, are reported to be metabolically quite stable (Russev et al., 1975). We have confirmed this result with bulk H2B-HA in our system (Supplemental Figure S2). This finding also suggests that the function of H2B polyubiquitylation is not linked to the degradation of excess histones (Gunjan and Verreault, 2003). We favor the idea that K48-linked H2B polyubiquitylation plays a nonproteolytic, regulatory role in some aspect of chromatin function. This notion is supported by our finding that the ubiquitylated forms of the $\mathrm{NK}+$ and coreK $+\mathrm{H} 2 \mathrm{~B}$ mutants are associated with chromatin (Figure 4) and is analogous to what has been observed for the Met4 transcription factor, where limited K48-linked poly-Ub chains modulate Met4 function without altering its stability (Flick et al., 2004; Flick et al., 2006). It is possible that multiple $\mathrm{Ub}$ chains on $\mathrm{H} 2 \mathrm{~B}$ recruit Ub-dependent chaperones, such as Cdc48/p97 and the base of the 19S proteasome (Jentsch and Rumpf, 2007), which can in turn alter chromatin structure. Recently, it was found that Cdc48/p49 can extract polyubiquitylated Aurora B kinase from chromatin (Ramadan et al., 2007). By analogy, perhaps polyubiquitylation of $\mathrm{H} 2 \mathrm{~B}$ is important for mediating its eviction from chromatin as part of nonreplicative histone H2B exchange (Jamai et al., 2007).

In addition to revealing ubiquitylation at noncanonical sites in H2B, our data also demonstrate that lysine 123 itself is subject to polyubiquitylation (Figure 2). Intriguingly, both mono- and polyubiquitylation at $\mathrm{K} 123$ is dependent on RAD6/BRE1 (Figure 2), revealing that the Rad6-Bre1 complex has selectivity for this residue and is the only ubiquitylation pathway that can target lysine 123. Given that many lysine residues on $\mathrm{H} 2 \mathrm{~B}$ can potentially serve as sites for $\mathrm{Ub}$ conjugation, the specificity between Rad6-Bre1 and K123 is striking and raises the issue of why other Ub-ligases fail to ubiquitylate K123. One possibility would be if the other Ub-ligases accessed a different pool of H2B than Rad6-Bre1, one in which K123 is not exposed in a context that is appropriate for ubiquitylation.

Regardless of how specificity between K123 and Rad6Bre1 is established, these findings raise the distinct possibility that the previously described functions of H2B-K123 ubiquitylation are mediated by poly- $\mathrm{Ub}$ chains. This is an important realization, because poly-Ub chains may be able to interact with protein factors that cannot bind tightly to a single Ub moiety (Jamai et al., 2007). As described above, poly-Ub chains could recruit $\mathrm{Ub}$-dependent chaperones to chromatin, which in turn facilitate the known functions of K123 ubiquitylation. Indeed, we have previously found that H2B ubiquitylation is required for recruitment of $19 \mathrm{~S}$ proteasomal ATPases to chromatin (Ezhkova and Tansey, 2004) and that mutations in these ATPases result in loss of histone H3 (K4 and K79) methylation, which is itself a Rad6-Bre1/ K123-dependent process. This finding has led us to propose that 195 proteins come to chromatin in a Ub-dependent manner and promote $\mathrm{H} 3$ methylation by altering local chromatin structure. One weakness in this model has been the fact that proteasome subunits have low affinity for mono-Ub
(Deveraux et al., 1994), making it difficult to imagine how monoubiquitylation of $\mathrm{H} 2 \mathrm{~B}$ at K123 could be linked to $19 \mathrm{~S}$ recruitment. The demonstration that $\mathrm{H} 2 \mathrm{~B}$ is polyubiquitylated at this site makes a direct link between Rad6-Bre1dependent ubiquitylation and 19S recruitment to chromatin more likely.

Our data also raise the possibility that transcriptional effects mediated by Ubp 8 and Ubp 10 could be a result of trimming poly-Ub chains, rather than deubiquitylating $\mathrm{H} 2 \mathrm{~B}$ entirely. It is difficult to dissect the relative contribution of mono- versus polyubiquitylation at lysine 123. Although the steady-state levels of polyubiquitylated H2B are considerably lower than the K123-monoubiquitylated form, this does not necessarily mean that $\mathrm{H} 2 \mathrm{~B}$ bearing a single $\mathrm{Ub}$ at $\mathrm{K} 123$ is the active species. Not only is ubiquitylation a highly dynamic process, but $\mathrm{H} 2 \mathrm{~B}$ polyubiquitylation may be spatially or temporally restricted, making it possible that H2B is extensively ubiquitylated at some sites in the genome where it the modification exerts its function. At this point, we do not know whether Rad6-Bre1 catalyzes K123-linked poly-Ub chains, or whether it primes $\mathrm{H} 2 \mathrm{~B}$ for polyubiquitylation by another ligase. We have found, however, that the $\mathrm{E} 4, \mathrm{Ufd} 2$, which is involved in $\mathrm{Ub}$-chain elongation (Koegl et al., 1999), is not required for polyubiquitylation at this site (data not shown), and we note that Rad6 can polyubiquitylate H2B in vitro (Sung et al., 1988), suggesting that perhaps Rad6-Bre1 are sufficient for driving Ub-chain formation at K123.

Our genetic data (Figure 5) show that, in the presence of the $\mathrm{K} 123+$ form of $\mathrm{H} 2 \mathrm{~B}$, disruption of either the Bre1 Ubligase or the $\mathrm{Ubp} 8 / \mathrm{Ubp} 10$ deubiquitylating enzymes blocks yeast cell viability. This result strongly suggests that both H2B ubiquitylation and deubiquitylation can be essential, supporting the notion that cycling of $\mathrm{H} 2 \mathrm{~B}$ ubiquitylation is a critical part of histone function (Emre and Berger, 2004). Whether this phenomenon reflects cycling of total K123 ubiquitylation, or essential trimming of the poly- $\mathrm{Ub}$ chain on K123, however, remains to be determined.

Are histones polyubiquitylated in other contexts? Early studies identified polyubiquitylated forms of both H2A and H2B (Davie et al., 1987, 1991), and found that both monoand polyubiquitylated $\mathrm{H} 2 \mathrm{~B}$ are preferentially localized to transcriptionally active chromatin domains (Nickel et al., 1989; Davie et al., 1991). It is thus possible that poly-Ub chains broadly regulate histone function in eukaryotic cells. The demonstration of H2B polyubiquitylation in a genetically tractable model organism should permit future analyses of the role these polyubiquitylation events play in control of chromatin dynamics.

\section{ACKNOWLEDGMENTS}

We thank G. Collins, A. Daulny, and A. Leung for helpful discussion and comments on the manuscript. For reagents we thank R. Deshaies, P. Kaiser, D. Finley, M.-A. Osley (University of New Mexico Health Sciences Center, Albuquerque, NM), and B. Stillman. W.P.T. was a Kimmel Foundation and Leukemia and Lymphoma Society Scholar. This work is supported by National Institutes of Health Grant GM067728.

\section{REFERENCES}

Bloom, J., Amador, V., Bartolini, F., DeMartino, G., and Pagano, M. (2003) Proteasome-mediated degradation of p21 via N-terminal ubiquitinylation. Cell 115, 71-82.

Crook, T., Ludwig, R. L., Marston, N. J., Willkomm, D., and Vousden, K. H. (1996). Sensitivity of p53 lysine mutants to ubiquitin-directed degradation targeted by human papillomavirus E6. Virology 217, 285-292. 
Daniel, J. A., Torok, M. S., Sun, Z. W., Schieltz, D., Allis, C. D., Yates, J. R., 3rd, and Grant, P. A. (2004). Deubiquitination of histone H2B by a yeast acetyltransferase complex regulates transcription. J. Biol. Chem. 279, 1867-1871.

Davie, J. R., Delcuve, G. P., Nickel, B. E., Moirier, R., and Bailey, G. (1987) Reduced levels of histones $\mathrm{H} 1 \mathrm{o}$ and $\mathrm{H} 1 \mathrm{~b}$, and unaltered content of methylated DNA in rainbow trout hepatocellular carcinoma chromatin. Cancer Res. 47 , 5407-5410

Davie, J. R., Lin, R., and Allis, C. D. (1991). Timing of the appearance of ubiquitinated histones in developing new macronuclei of Tetrahymena thermophila. Biochem. Cell Biol. 69, 66-71.

de Napoles, M. et al. (2004). Polycomb group proteins Ring1A/B link ubiquitylation of histone H2A to heritable gene silencing and X inactivation. Dev. Cell 7, 663-676.

Dehe, P. M., Pamblanco, M., Luciano, P., Lebrun, R., Moinier, D., Sendra, R., Verreault, A., Tordera, V., and Geli, V. (2005). Histone H3 lysine 4 monomethylation does not require ubiquitination of histone H2B. J. Mol. Biol. 353, $477-484$

Deveraux, Q., Ustrell, V., Pickart, C., and Rechsteiner, M. (1994). A 26 S protease subunit that binds ubiquitin conjugates. J. Biol. Chem. 269, 7059-7061.

Dohmen, R. J., Stappen, R., McGrath, J. P., Forrova, H., Kolarov, J., Goffeau, A., and Varshavsky, A. (1995). An essential yeast gene encoding a homolog of ubiquitin-activating enzyme. J. Biol. Chem. 270, 18099-18109.

Dover, J., Schneider, J., Tawiah-Boateng, M. A., Wood, A., Dean, K., Johnston, M., and Shilatifard, A. (2002). Methylation of histone H3 by COMPASS requires ubiquitination of histone H2B by Rad6. J. Biol. Chem. 277, 28368-28371.

Emre, N. C., and Berger, S. L. (2004). Histone H2B ubiquitylation and deubiquitylation in genomic regulation. Cold Spring Harb. Symp. Quant. Biol. 69 289-299.

Emre, N. C. et al. (2005). Maintenance of low histone ubiquitylation by Ubp10 correlates with telomere-proximal Sir2 association and gene silencing. Mol. Cell 17, 585-594.

Ezhkova, E., and Tansey, W. P. (2004). Proteasomal ATPases link ubiquitylation of histone H2B to methylation of histone H3. Mol. Cell 13, 435-442.

Fang, J., Chen, T., Chadwick, B., Li, E., and Zhang, Y. (2004). Ring1b-mediated $\mathrm{H} 2 \mathrm{~A}$ ubiquitination associates with inactive $\mathrm{X}$ chromosomes and is involved in initiation of X inactivation. J. Biol. Chem. 279, 52812-52815.

Flick, K., Ouni, I., Wohlschlegel, J. A., Capati, C., McDonald, W. H., Yates, J. R., and Kaiser, P. (2004). Proteolysis-independent regulation of the transcription factor Met4 by a single Lys 48-linked ubiquitin chain. Nat. Cell Biol. 6, 634-641.

Flick, K., Raasi, S., Zhang, H., Yen, J. L., and Kaiser, P. (2006). A ubiquitininteracting motif protects polyubiquitinated Met4 from degradation by the $26 \mathrm{~S}$ proteasome. Nat. Cell Biol. 8, 509-515.

Gardner, R. G., Nelson, Z. W., and Gottschling, D. E. (2005). Ubp10/Dot4p regulates the persistence of ubiquitinated histone $\mathrm{H} 2 \mathrm{~B}$ : distinct roles in telomeric silencing and general chromatin. Mol. Cell. Biol. 25, 6123-6139.

Goldknopf, I. L., and Busch, H. (1977). Isopeptide linkage between nonhistone and histone 2A polypeptides of chromosomal conjugate-protein A24. Proc. Natl. Acad. Sci. USA 74, 864-868.

Goldknopf, I. L., Taylor, C. W., Baum, R. M., Yeoman, L. C., Olson, M. O. Prestayko, A. W., and Busch, H. (1975). Isolation and characterization of protein A24, a "histone-like" non-histone chromosomal protein. J. Biol. Chem. 250, 7182-7187.

Gunjan, A., and Verreault, A. (2003). A Rad53 kinase-dependent surveillance mechanism that regulates histone protein levels in S. cerevisiae. Cell 115, $537-549$.

Henry, K. W., Wyce, A., Lo, W. S., Duggan, L. J., Emre, N. C., Kao, C. F., Pillus, L., Shilatifard, A., Osley, M. A., and Berger, S. L. (2003). Transcriptional activation via sequential histone $\mathrm{H} 2 \mathrm{~B}$ ubiquitylation and deubiquitylation, mediated by SAGA-associated Ubp8. Genes Dev. 17, 2648-2663.

Hodgins, R. R., Ellison, K. S., and Ellison, M. J. (1992). Expression of a ubiquitin derivative that conjugates to protein irreversibly produces phenotypes consistent with a ubiquitin deficiency. J. Biol. Chem. 267, 8807-8812.

Hwang, W. W., Venkatasubrahmanyam, S., Ianculescu, A. G., Tong, A., Boone, C., and Madhani, H. D. (2003). A conserved RING finger protein required for histone H2B monoubiquitination and cell size control. Mol. Cell $11,261-266$.

Jamai, A., Imoberdorf, R. M., and Strubin, M. (2007). Continuous histone H2B and transcription-dependent histone $\mathrm{H} 3$ exchange in yeast cells outside of replication. Mol. Cell 25, 345-355.

Jentsch, S., and Rumpf, S. (2007). Cdc48 (p97): a "molecular gearbox" in the ubiquitin pathway? Trends Biochem. Sci. 32, 6-11.
Jenuwein, T., and Allis, C. D. (2001). Translating the histone code. Science 293, 1074-1080.

Kahana, A., and Gottschling, D. E. (1999). DOT4 links silencing and cell growth in Saccharomyces cerevisiae. Mol. Cell. Biol. 19, 6608-6620.

Kaiser, P., and Tagwerker, C. (2005). Is this protein ubiquitinated? Methods Enzymol. 399, 243-248.

Kao, C. F., Hillyer, C., Tsukuda, T., Henry, K., Berger, S., and Osley, M. A (2004). Rad6 plays a role in transcriptional activation through ubiquitylation of histone H2B. Genes Dev. 18, 184-195.

Knop, M., Siegers, K., Pereira, G., Zachariae, W., Winsor, B., Nasmyth, K., and Schiebel, E. (1999). Epitope tagging of yeast genes using a PCR-based strategy: more tags and improved practical routines. Yeast 15, 963-972.

Koegl, M., Hoppe, T., Schlenker, S., Ulrich, H. D., Mayer, T. U., and Jentsch, S. (1999). A novel ubiquitination factor, $\mathrm{E} 4$, is involved in multiubiquitin chain assembly. Cell 96, 635-644.

Levinger, L., and Varshavsky, A. (1982). Selective arrangement of ubiquitinated and D1 protein-containing nucleosomes within the Drosophila genome. Cell 28, 375-385.

Li, B., Howe, L., Anderson, S., Yates, J. R., 3rd, and Workman, J. L. (2003). The Set2 histone methyltransferase functions through the phosphorylated CTD of RNA polymerase II. J. Biol. Chem. 278, 8897-8903.

Liang, C., and Stillman, B. (1997). Persistent initiation of DNA replication and chromatin-bound MCM proteins during the cell cycle in cdc6 mutants. Genes Dev. 11, 3375-3386.

Muratani, M., Kung, C., Shokat, K. M., and Tansey, W. P. (2005). The F box protein Dsg1/Mdm30 is a transcriptional coactivator that stimulates Gal4 turnover and cotranscriptional mRNA processing. Cell 120, 887-899.

Muratani, M., and Tansey, W. P. (2003). How the ubiquitin-proteasome system controls transcription. Nat. Rev. Mol. Cell Biol. 4, 192-201.

$\mathrm{Ng}$, H. H., Dole, S., and Struhl, K. (2003). The Rtf1 component of the Paf1 transcriptional elongation complex is required for ubiquitination of histone H2B. J. Biol. Chem. 278, 33625-33628.

Nickel, B. E., Allis, C. D., and Davie, J. R. (1989). Ubiquitinated histone H2B is preferentially located in transcriptionally active chromatin. Biochemistry $28,958-963$

Osley, M. A. (2004). H2B ubiquitylation: the end is in sight. Biochim. Biophys. Acta $1677,74-78$

Pickart, C. M., and Fushman, D. (2004). Polyubiquitin chains: polymeric protein signals. Curr. Opin. Chem. Biol. 8, 610-616.

Ramadan, K., Bruderer, R., Spiga, F. M., Popp, O., Baur, T., Gotta, M., and Meyer, H. H. (2007). Cdc48/p97 promotes reformation of the nucleus by extracting the kinase Aurora B from chromatin. Nature 450, 1258-1262.

Recht, J., and Osley, M. A. (1999). Mutations in both the structured domain and $\mathrm{N}$-terminus of histone H2B bypass the requirement for Swi-Snf in yeast. EMBO J. 18, 229-240.

Robzyk, K., Recht, J., and Osley, M. A. (2000). Rad6-dependent ubiquitination of histone H2B in yeast. Science 287, 501-504.

Russev, G., Anachkova, B., and Tsanev, R. (1975). Fractionation of rat-liverchromatin nonhistone proteins into two groups with different metabolic rates. Eur. J. Biochem. 58, 253-257.

Ruthenburg, A. J., Li, H., Patel, D. J., and Allis, C. D. (2007). Multivalent engagement of chromatin modifications by linked binding modules. Nat. Rev Mol. Cell Biol. 8, 983-994.

Salghetti, S. E., Muratani, M., Wijnen, H., Futcher, B., and Tansey, W. P. (2000). Functional overlap of sequences that activate transcription and signal ubiquitin-mediated proteolysis. Proc. Natl. Acad. Sci. USA. 97, 3118-3123.

Shahbazian, M. D., Zhang, K., and Grunstein, M. (2005). Histone H2B ubiquitylation controls processive methylation but not monomethylation by Dot1 and Set1. Mol. Cell 19, 271-277.

Sun, Z. W., and Allis, C. D. (2002). Ubiquitination of histone H2B regulates H3 methylation and gene silencing in yeast. Nature 418, 104-108.

Sung, P., Prakash, S., and Prakash, L. (1988). The RAD6 protein of Saccharomyces cerevisiae polyubiquitinates histones, and its acidic domain mediates this activity. Genes Dev. 2, 1476-1485.

Wood, A. et al. (2003a). Bre1, an e3 ubiquitin ligase required for recruitment and substrate selection of rad6 at a promoter. Mol. Cell 11, 267-274.

Wood, A., Schneider, J., Dover, J., Johnston, M., and Shilatifard, A. (2003b). The Paf1 complex is essential for histone monoubiquitination by the Rad6/ Bre1 complex, which signals for histone methylation by COMPASS and Dot1p. J. Biol. Chem. 278, 34739-34742. 
Wood, A., Schneider, J., Dover, J., Johnston, M., and Shilatifard, A. (2005). The Bur1/Bur2 complex is required for histone $\mathrm{H} 2 \mathrm{~B}$ monoubiquitination by Rad6/Bre1 and histone methylation by COMPASS. Mol. Cell 20, 589-599.

Wyce, A., Xiao, T., Whelan, K. A., Kosman, C., Walter, W., Eick, D., Hughes, T. R., Krogan, N. J., Strahl, B. D., and Berger, S. L. (2007). H2B ubiquitylation acts as a barrier to Ctk1 nucleosomal recruitment prior to removal by $\mathrm{Ubp} 8$ within a SAGA-related complex. Mol. Cell 27, 275-288.

Xiao, T., Kao, C. F., Krogan, N. J., Sun, Z. W., Greenblatt, J. F., Osley, M. A., and Strahl, B. D. (2005). Histone H2B ubiquitylation is associated with elongating RNA polymerase II. Mol. Cell Biol. 25, 637-651.

Yaglom, J., Linskens, M. H., Sadis, S., Rubin, D. M., Futcher, B., and Finley, D. (1995). p34Cdc28-mediated control of Cln3 cyclin degradation. Mol. Cell. Biol. 15, 731-741.

Yamashita, K., Shinohara, M., and Shinohara, A. (2004). Rad6-Bre1-mediated histone H2B ubiquitylation modulates the formation of double-strand breaks during meiosis. Proc. Natl. Acad. Sci. USA 101, 11380-11385. 